\title{
Spiral Galaxies Rotation Curves With Renormalization Group Effects to Gravity
}

\author{
Paulo L. C. de Oliveira* \\ Departamento de Física, Universidade Federal do Espírito Santo \\ E-mail: paulo_lco@yahoo.com.br
}

\begin{abstract}
In this work we explore the Renormalization Group (RG) corrections to General Relativity in the galactic domain. The approach here employed, which we call RGGR, is based on a relation between the RG energy scale $\mu$ and the Newtonian potential $\Phi_{N}: \mu \propto \Phi_{N}^{\alpha}$, where $\alpha$ is a constant. Here we present the fits to 3 of a larger sample of galaxies (about 50), with which we confirm some results: RGGR without dark matter generates galaxy rotation curves that are usually better than the ones generated by either MOND or NFW (once both the shape and the expected massto-light ratios are evaluated). Generally, galaxies that have clear problems with the NFW halo, due to its cuspy nature, are nicely fit within RGGR.
\end{abstract}

VIII International Workshop on the Dark Side of the Universe,

June 10-15, 2012

Rio de Janeiro, Brazil

${ }^{*}$ Speaker. 
In this work we explore the RGGR model, where quantum corrections to General Relativity can reproduce the observed rotation curves of galaxies with significantly less or even no dark matter at all, as the case we explore here. It was shown in $[1,2]$ that a RG equation to the running of the gravitational coupling constant leads to a variation of $\mathrm{G}$ with a energy scale $\mu$, with the form

$$
G(\mu)=\frac{G_{0}}{1+v \ln \left(\frac{\mu^{2}}{\mu_{0}^{2}}\right)}=G_{0}\left(1-2 v \ln \frac{\mu}{\mu_{0}}+O\left(v^{2}\right)\right),
$$

where $v \approx 10^{-7}$ is the parameter associated to the quantum corrections. The weak field limit can be found from the equations of motion obtained with the variation of the Einstein-Hilbert action with the metric field, but considering $\mathrm{G}$ as a variable, leading to a modified potential. The next step in the modeling is the choice of an appropriate energy scale to the analysis of the rotation curves. The most natural choice is the Newtonian potential $\Phi_{N}$, so we generalize it to [1]

$$
\frac{\mu}{\mu_{0}}=\left(\frac{\Phi_{N}}{\Phi_{0}}\right)^{\alpha}
$$

where $\alpha$ and $\Phi_{0}$ are constants, the latter one being physically unimportant while the first one is fitted to each galaxy and expected to be related with the its mass. With the above expressions the circular velocity of the galaxy is

$$
V^{2}(R)=V_{N}^{2}\left(1-\frac{\alpha v c^{2}}{\Phi_{N}}\right)
$$

We have analysed the rotation curves of around 50 spiral galaxies described in the literature [3 -5], spanning a large range of sizes and masses. We fit the data with the usual $\chi^{2}$ statistics, and for comparison we also fit the NFW profile for dark matter and the Modified Newtonian Dynamics (MOND) approach. Our results show that the RGGR model fits the galaxies better than MOND, and it is also competitive with the NFW dark matter profiles, with one less free parameter. We show below one rotation curve from each of the samples presented in [3-5] respectively, where in each plot the yellow curve is the stellar contribution, the purple curve is the gas one, the green is the total baryonic curve and the black is the RGGR curve. In the case of NGC 5055 a bulge-disk decomposition is made and the yellow and red curves represents the bulge and disk respectively.

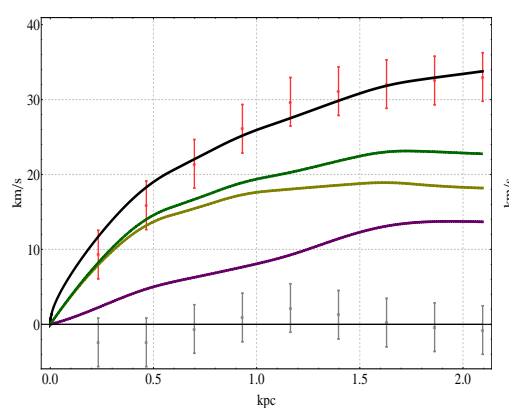

(a) NFW: $\chi_{v}^{2}=0.32, \gamma=7.48$

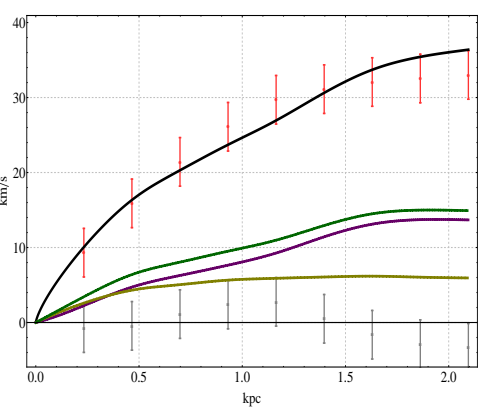

(b) MOND: $\chi_{v}^{2}=0.45, \gamma=0.80$

Figure 1: UGC 7559

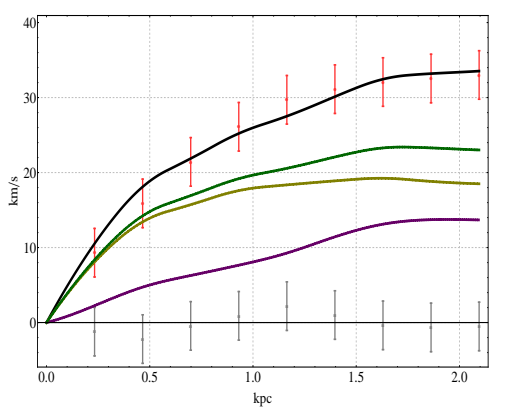

(c) RGGR: $\chi_{v}^{2}=0.19, \gamma=7.73$ 


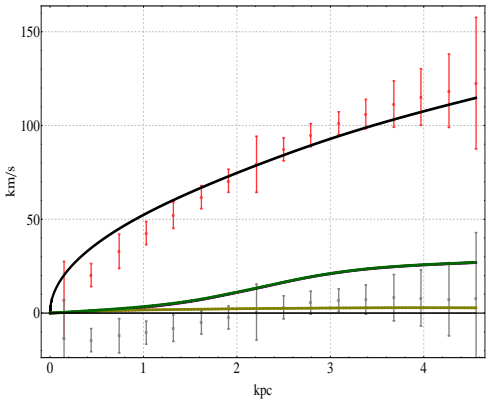

(a) NFW: $\chi_{v}^{2}=1.31, \gamma=0.01$

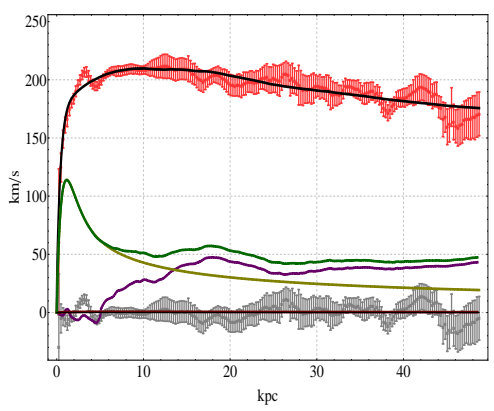

(a) $\chi_{v}^{2}=0.56, \gamma_{B}=0.21, \gamma_{D}=0$

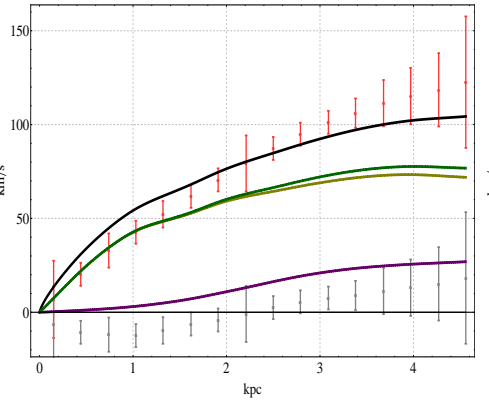

(b) MOND: $\chi_{v}^{2}=1.25, \gamma=4.80$

Figure 2: UGC 4325

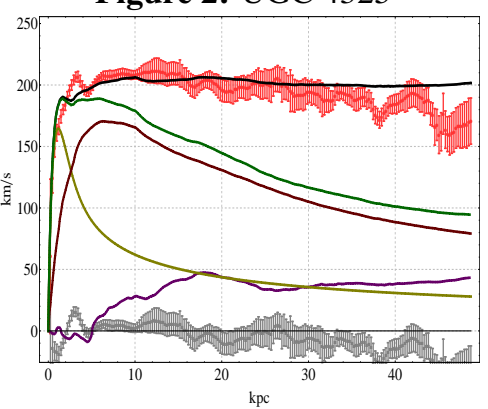

(b) $\chi_{v}^{2}=2.14, \gamma_{B}=0.43, \gamma_{D}=0.45$

Figure 3: NGC 5055

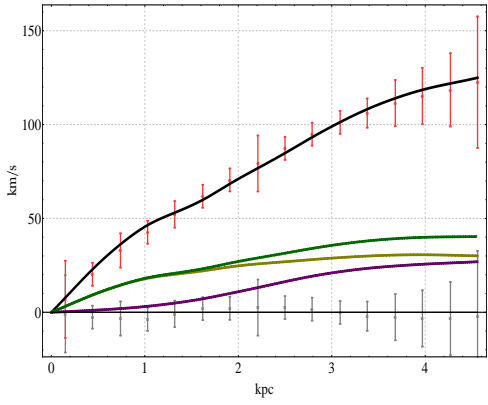

(c) RGGR: $\chi_{v}^{2}=0.10, \gamma=0.84$

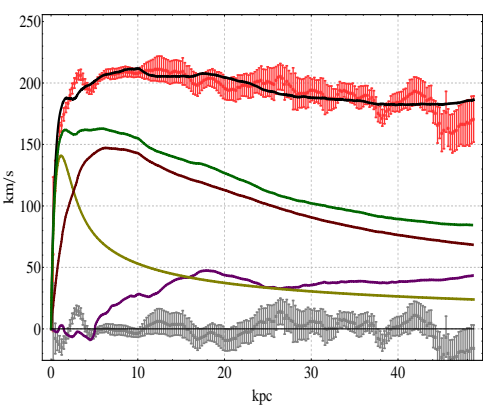

(c) $\chi_{v}^{2}=0.88, \gamma_{B}=0.32, \gamma_{D}=0.33$

In the plots above we display the best fit values for the mass-to-light ratios $\gamma$ (or $\gamma_{B}, \gamma_{D}$ for bulge and disk of NGC 5055), in units of solar mass/luminosity (in the R band for UGCs 7559 and 4325 and in the Spitzer $3.6 \mathrm{~mm}$ band for NGC 5055), and the value of the reduced $\chi^{2}$. Currently we are searching for the possible correlations between $\alpha$ and galactic parameters such as baryonic mass, maximal velocity and others, in order to probe the nature of $\alpha$. We also expect to relate the model predictions with others, such as the Universal Rotation Curve [6] and MOND.

The author would like to thank profs. Júlio Fabris, Davi Rodrigues, Paolo Salucci and Gianfranco Gentile for the comments and discussions, and CAPES for financial support.

\section{References}

[1] D. C. Rodrigues, P. S. Letelier, I. L. Shapiro, Galaxy rotation curves from General Relativity with Renormalization Group corrections, JCAP 04 (2010) 020 [astro-ph.CO/0911.4967].

[2] I.L. Shapiro, J. Solà and H. Stefancic, Running $G$ and $\Lambda$ at low energies from physics at $M_{X}$ : possible cosmological and astrophysical implications, JCAP 01 (2005) 012 [hep-ph/0410095].

[3] W. J. G. de Blok, A. Bosma, High-resolution rotation curves of Low Surface Brightness galaxies, $A \& A 385$ (2002) 816.

[4] R. A. Swaters et. al., Are Dwarf Galaxies Dominated by Dark Matter?, ApJ 729 (2011) 118 [astro-ph.CO/1101.3120v1].

[5] W. J. G. de Blok et al., High-Resolution Rotation Curves and Galaxy Mass Models from THINGS, AJ 136 (2008) 2648 [astro-ph/0810.2100].

[6] P. Salucci et al., The Universal Rotation Curve of Spiral Galaxies II The Dark Matter Distribution out to the Virial Radius, MNRAS 378 (2007) 41 [astro-ph/0703115]. 